\title{
Of the bugs that shape us: maternal obesity, the gut microbiome, and long-term disease risk
}

\author{
Wajiha Gohir', Elyanne M. Ratcliffe ${ }^{2}$ and Deborah M. Sloboda ${ }^{1,2,3}$
}

Chronic disease risk is inextricably linked to our early-life environment, where maternal, fetal, and childhood factors predict disease risk later in life. Currently, maternal obesity is a key predictor of childhood obesity and metabolic complications in adulthood. Although the mechanisms are unclear, new and emerging evidence points to our microbiome, where the bacterial composition of the gut modulates the weight gain and altered metabolism that drives obesity. Over the course of pregnancy, maternal bacterial load increases, and gut bacterial diversity changes and is influenced by pre-pregnancy-and pregnancy-related obesity. Alterations in the bacterial composition of the mother have been shown to affect the development and function of the gastrointestinal tract of her offspring. How these microbial shifts influence the maternal-fetal-infant relationship is a topic of hot debate. This paper will review the evidence linking nutrition, maternal obesity, the maternal gut microbiome, and fetal gut development, bringing together clinical observations in humans and experimental data from targeted animal models.

E ach year 36 million people die due to chronic diseases. Chronic disease risk has been typically ascribed to arise from either genetic or lifestyle factors. Although the rise in the occurrence of obesity to the epidemic proportions that we now observe worldwide (1) can no longer be solely attributed to genetic factors. Furthermore, the difficulty in reducing the rising rate of obesity and its comorbidities with lifestyle changes has resulted in the exploration of other pathways that may contribute to disease risk. There is no doubt that obesity is the result of complex, multifaceted causal pathways and the quest for a single magic bullet is becoming a thing of the past.

The developing organism is plastic, where genotype responses to a changing environment have led to the hypothesis that disease risk is the result of complex gene-environment interactions (2). Of critical importance, is the recognition that our "environment" includes the period encompassing the developmental milieu within which gametes, germ/stem, and somatic cells will take shape and differentiate into established organ systems. It is proposed that the inherent developmental plasticity of an organism during critical windows of development allows it to respond to cues that will ultimately determine the mature (adult) phenotype; under some circumstances, these adaptations may lead to increased disease risk (3). In this regard, epidemiological and experimental data have inextricably shown a relationship between the in utero environment and the risk of developing chronic disease later in life (3). A multitude of "modifying" cues inducing developmental adaptations that impact on disease risk have been identified $(4,5)$. Most recently, research into the gut microbiota has become one of most studied factors influencing disease risk. The gut microbiota is modulated by several factors such as diet, age, and health status (6). It is now thought that the responses of commensal bacteria, residing symbiotically within our gut, to changing nutritional/endocrine/infectious stimuli likely contribute to long-term metabolic dysfunction, obesity, and chronic disease risk. In this review, we consider the changes that occur in the maternal gut microbiome over the course of pregnancy, whether maternal gut microbiome is influenced by maternal obesity and finally the impact of maternal nutrition on the development of the fetal gut microbiome.

\section{OBESITY AND THE GUT MICROBIOME: GENERAL OVERVIEW}

The relationship between our gut microbiome and metabolic and obesogenic risk has been reviewed extensively (7) and will not be reviewed here. However, some description of the relationship between commensal bacteria and mammalian metabolic function is required in order to understand how the early-life environment may modulate host-bacterial impacts on physiological function.

The successful establishment of commensal gut microbiota is essential for metabolizing indigestible polysaccharides, producing essential nutrients, and protecting against the colonization of pathogenic bacteria in the mammalian gut (8). Critically, gut bacterial populations also play a critical role in regulating fat storage-where studies have shown that colonizing germ-free mice with microbiota extracted from conventional mice induces a dramatic increase in body fat, despite a decrease in food consumption, through de novo lipogenesis (9). Even more convincing was the demonstration that colonization of germ-free mice with microbiota from 


\section{Maternal obesity and gut microbiome}

both genetically obese and diet-induced obese mice results in a greater increase in body fat than when colonized with microbiota from lean mice or mice fed a low-fat diet (10). How this shift in adipose tissue accumulation occurs is the focus of much research. Different bacterial populations use and produce different nutritional substrates and metabolites, and have varying capacities for energy harvest. Of these populations, the two most abundant phyla in the mammalian gut, Firmicutes and Bacteroidetes have received the most attention (10-12). Genetically obese $o b / o b$ mice demonstrate shifts in the proportions of Firmicutes and Bacteroidetes where Bacteroidetes were significantly reduced, while Firmicutes were increased in abundance in obese mice relative to lean mice (12). Consistent with this, shotgun sequencing of the gut microbiome of obese mice demonstrates enrichment in genes encoding for enzymes involved in energy extraction, supporting the concept that these bacterial populations allow obese mice to harvest more energy from their diet (10).

Such observations involving fluctuations in the proportions of bacteria populations are not limited to animal models. Analysis of the core gut microbiome in obese and lean twins by sequencing of the $16 \mathrm{~S}$ rRNA gene sequence has shown that obesity is associated with a decrease in abundance of Bacteroidetes and altered expression of genes involved in metabolic pathways that favor increased energy uptake and adipogenesis (13). Nutrition manipulation in obese individuals through fat-restricted or carbohydrate-restricted diets showed an increased shift in Bacteroidetes and a decrease in Firmicutes populations over time and these shifts were correlated with a loss of body weight (11) suggesting that these bacterial populations may be calorically responsive (11). Although other studies support the relationship between Bacteroidetes and energy intake (14), others do not (15), highlighting the importance of looking beyond phylum-level changes in bacteria and rather understanding metagenomic changes may prove to be more illuminating (16). Finally, given the fact that gut microbiota both yield energy for their own growth and produce end products such as short chain fatty acids (SCFAs) for host metabolism (17) suggests that metabolomics are also required to understand the relationship between gut microbiome, obesity, and metabolic dysfunction. The metabolic activity of the gut microbiota is increased in obese children compared with lean children, thus gut dysbiosis is not limited to obesity in adulthood; it has also involved in the etiology of childhood obesity (18). As in the adult literature, there is evidence to suggest that not only are there shifts in fecal microbial populations in overweight children, but also variations in bacteria exist at the species levels (19).

Thus, it is now clear that the gut microbiome is a contributor to the weight gain and altered metabolism that accompanies obesity $(12,20)$. Gut microbiota affect host metabolism by modulating energy extraction (10), immunity (21), and lipid metabolism (20), where both dietary factors (22) and genetics (12) influence gut microbial composition (23) and interactions between the gut microbiome and immunity likely contribute to diseases with aberrant inflammation, including obesity.

\section{MATERNAL OBESITY}

It is not surprising that as our knowledge of the microbialhost relationship with metabolic outcomes evolves, that more attention is being paid to the maternal gut microbiome and its association with maternal obesity and childhood outcome. Maternal obesity is currently one of the leading factors predicting childhood obesity (24). Maternal obesity is associated with abnormal feto-placental function (25), offspring obesity risk (24), and increased disease risk in general. Although maternal high-fat (HF) diet models in animals have made significant advances in our understanding of the underlying mechanisms, linking maternal obesity with offspring obesity and metabolic dysfunction which one (or combination) of the identified signaling pathways is best for targeted interventions to reduce childhood and adolescent obesity and metabolic compromise is as of yet, unclear.

Impacts of Maternal Obesity: Overview of Epidemiologic Studies Obese women are at risk of a multitude of pregnancy complications, including increased risk of miscarriage, gestational diabetes, hypertension, and preeclampsia as well as increased risk of C-section delivery (26). It has been reported that the incidence of both preeclampsia and gestational hypertension increases linearly with increasing maternal BMI and that morbidly obese women have a threefold higher risk of experiencing obstetrical complications (Odds ratio (OR) 4.21; 95\% confidence interval (CI) 3.45, 5.13 preeclampsia and OR 3.66; 95\% CI 3.23, 4.15 for gestational hypertension) (27). Maternal obesity is also associated with significant changes in endocrine and metabolic function where circulating levels of SCFA (28) as well as pro-inflammatory cytokines (29) are elevated in mothers that are obese during pregnancy.

Maternal obesity not only impacts the mother's health during gestation, but also has both short- and long-term ramifications for her offspring (30). Both maternal obesity and elevated gestational weight gain during pregnancy is associated with fetal macrosomia. The incidence of high birth weight increases linearly with increasing maternal BMI and, relative to a reference group, both obese and morbidly obese women show a twofold greater risk of delivering a baby weighing $>4000 \mathrm{~g}$ (OR 2.01 (95\% CI 1.83, 2.22) and OR 2.62 (95\% CI 2.30, 2.98), respectively) (27). Catalano et al. found fetuses of obese mothers had greater percent body fat and developed insulin resistance already in utero (31). Neural tube defects and other developmental anomalies are more common in infants born to obese women (32).

Maternal obesity affects the placenta. Obese women are more likely to have larger placentas and larger placenta to birth weight ratio: across the overweight to morbidly obese BMI range, women were progressively less likely to have a small placenta and more likely to have a large placenta and overweight, with a lower "placental efficiency" than normal reference groups (27). Recent data suggest that maternal BMI is associated with activation of placental inflammatory pathways, and that activation of pro-inflammatory markers MCP-1 and tumor necrosis factor-alpha suggests a link between elevated 
pro-inflammatory cytokines in maternal plasma and activation of placental inflammatory pathways potentially influencing placental function (29). Consistent with this, others report that placentas from obese women develop exaggerated inflammatory responses with increased macrophage infiltration and pro-inflammatory cytokines (33) and this pro-inflammatory environment may have the capacity to modulate placental nutrient transfer. Placentas of obese women demonstrate decreased sodium-dependent neutral amino acid transporter (System A) expression (34) and, at least in vitro, IL-1 $\beta$ inhibited insulin-stimulated System A amino acid uptake in human placental trophoblast cells (35). Obese women show placental dysregulation of redox balance (36), decreased fatty acid transport, and increased placental lipoprotein lipase activity which could be responsible for placental triglycertides accumulation (37), although this contrasts to placenta-derived data from animal models studying diet-induced maternal obesity (see below).

Maternal obesity and excess gestational weight gain are associated with childhood obesity and increased incidence of metabolic syndrome (38). Indeed, macrosomia itself is associated with an increased risk of developing obesity and metabolic syndrome (39) which appears to persist for multiple generations (40). Finally, all cause mortality has been shown to be increased in offspring of obese mothers $(\mathrm{BMI}>30)$ compared with mothers with normal BMI (after adjustment for confounding variables; hazard ratio $1.35,95 \%$ CI 1.17-1.55) (41).

\section{Overview of Experimental Studies on Outcomes After Maternal Obesity}

Animal models have been used to study the effects of maternal obesity and pinpoint specific mechanisms that are involved. The outcome of maternal diet-induced obesity in animal models has been reviewed elsewhere (42) and will only be briefly reviewed here. In rodents, maternal HF diet during pregnancy and lactation induces obesity and hepatic steatosis in the offspring, irrespective of postnatal diet (43) and induces insulin and leptin resistance (44), hypertension (45), and hepatic inflammation in offspring (46). Sex-specific obesity risk and metabolic dysfunction have also been shown and have become a focus for some studies (47). Diet-induced maternal obesity in rodents induces sex-specific effects on placental growth and fetal metabolic phenotype (48), gene expression, and epigenetic regulation (49) — consistent with human data (50).

Fetuses from diet-induced obese ewes are macrosomic, hyperglycemic, and hyperinsulinemic (51) and show decreased insulin signaling in skeletal muscle associated with increased intramuscular triglycerides and higher expression of fatty acid transporters (52). In mice, diet-induced obesity during pregnancy was associated with inhibition of placental mammalian target of rapamycin signaling and reduced placental efficiency (53) and increased transplacental transport of nutrients such as glucose and neutral amino acids, resulting in increased fetal growth (54). In sheep, maternal obesity results in enhanced placental content of fatty acid transport proteins and transcription factors that regulate lipid metabolism (PPAR $\gamma)(55)$, increased levels of Toll-like receptors (TLR) 2 and TLR-4, macrophage markers and pro-inflammatory cytokines as well as upstream transcription factors in signaling pathways associated with inflammation (56).

\section{MATERNAL GUT MICROBIOME}

The concept that maternal gut bacterial populations change over the course of pregnancy to influence maternal and fetal metabolic development is novel and will no doubt expand the way we view maternal adaptation to pregnancy. Although it is well established that the mother makes critical metabolic, cardiovascular, endocrine, and skeletomuscular changes to accommodate the growth of the placenta and the fetus, there are few data describing pregnancy-associated changes in maternal gut microbiome. Maternal gut bacterial load increases over the course of pregnancy (57). There is a significant change in gut microbiome composition from the first to the third trimester of pregnancy, accompanied by increased bacterial diversity between mothers (58). Although the composition and diversity of the gut microbiome during the first trimester is similar to that of non-pregnant healthy women, over the course of pregnancy, there is an increase in the abundance of Proteobacteria and Actinobacteria (58). Since an increase in Proteobacteria has been observed in inflammatory bowel disease, it has been suggested that a similar dysbiosis of the gut occurs during the third trimester of pregnancy (59). Pro-inflammatory cytokines (such as IL-6 and tumor necrosis factor-alpha) are notably increased in stool samples collected from women during the third trimester in comparison to the first trimester (58). Germ-free mice inoculated with third trimester microbiota show signs of increased inflammation and greater adipocity; similar results has been observed in germfree mice that are inoculated with microbiota from an obese individual (58). Together, these observations suggest that these bacteria population shifts in the final trimester of pregnancy are associated with known changes in insulin resistance and inflammation that accompany normal pregnancy progression.

Although there is significant remodeling of the maternal gut microbiota from the first to third trimester, the microbiome appears to remain stable during the postnatal period $(58,60)$. Koren et al. reported that increased diversity persists in maternal microbiota up to 1 mo postpartum. Third trimester and 1 mo postpartum fecal samples were both enriched in Streptococcus (58). These data were supported by Jost et al., who investigated gut microbiota during the perinatal period. They found that the maternal gut microbiome was dominated by the Firmicutes phylum, Bacteroides, and Bifidobacterium and their density did not vary during the perinatal period (60). Thus, it appears that the maternal gut does not immediately revert to its non-pregnant state post delivery during the lactation period. These persistent pregnancy-like microbial populations may serve to faciliate (or be manipulated by) maternal adapation to the new lactational environment, although there are no data describing how maternal gut microbiota during lactation influences maternal physiological function.

The role of the maternal gut microbiome in modulating maternal obesity-induced changes in offspring metabolism 
is likely, since bacterial composition is critically dependent on nutrition (61). Since the bacterial composition of the gut has been proposed to modulate weight gain, impair metabolism resulting in obesity (62) through its regulation of energy extraction (10), immunity (21), and lipid metabolism (62), it seems likely that the composition of the maternal gut microbiome would impart downstream effects on pregnancy outcomes through effects on (i) maternal adaptation to pregnancy (ii) placental function, and (iii) the fetal intrauterine environment. It is proposed that a healthy maternal gut microbiota is modulated over the course of pregnancy (Figure 1a). Disrupted maternal gut microbiota such as in the case of maternal obesity, before pregnancy, is either amplified or further modified with pregnancy adaptations (Figure $\mathbf{1 b}$ ). These modifications lead to aberrant intrauterine environment that could lead to fetal poor/altered gut development and mediated increased chronic disease risk.

The intestinal microbiota composition during pregnancy is affected by pre-pregnancy weight and weight gain over the course of pregnancy. In a 2010 study, gut microbiota composition during the first and third trimester was examined in 36 normal weight women $(\mathrm{BMI}<25)$ and 18 overweight women (BMI > 30). Overweight pregnant women were found to have significantly higher levels of Bacteroides and Staphylococcus, where pregnancy weight gain was correlated with Bacteroides number (57). In contrast, Santacruz et al. detected an increase in Staphylococcus, Enterobacteriaceae, and Escherichia coli in overweight pregnant women and a decrease in Bifidobacterium and Bacteroides (63), not dissimilar to observations made in non-pregnant obese individuals. Although maternal overweight and excess weight gain during pregnancy causes aberrant gut microbiota, distinctly different from that of normal weight pregnant women, little is known about the impact of this dysbiosis on maternal or fetal metabolism during pregnancy. Priyadarshini et al. have shown an association between maternal SCFA levels and metabolic parameters where serum acetate levels were associated with maternal weight gain (28). SCFA are one of the major metabolites produced by gut microbiota during food fermentation, and levels of SCFAs depend on the composition of the gut microbiota (64). In fact, evidence that SCFAs (particularly propionate and butyrate) contribute to inflammatory and metabolic disorders including obesity (64) suggests that obesity-induced changes in the pregnant gut microbiota may impart changes in metabolic pathways via SCFA production (28). Studies looking at the effects of probiotic intake during pregnancy also provide evidence for a maternal microbiome-metabolism relationship. Laitinen et al. found that diet consultation along with probiotics during pregnancy improved maternal glucose tolerance and insulin sensitivity (65). Intriguingly, probiotics and dietary counseling have also been associated with reduced risk of gestational diabetes mellitus (66).

Thus, it is apparent that distinct differences in the maternal microbiome occur over pregnancy (58). Maternal weight gain
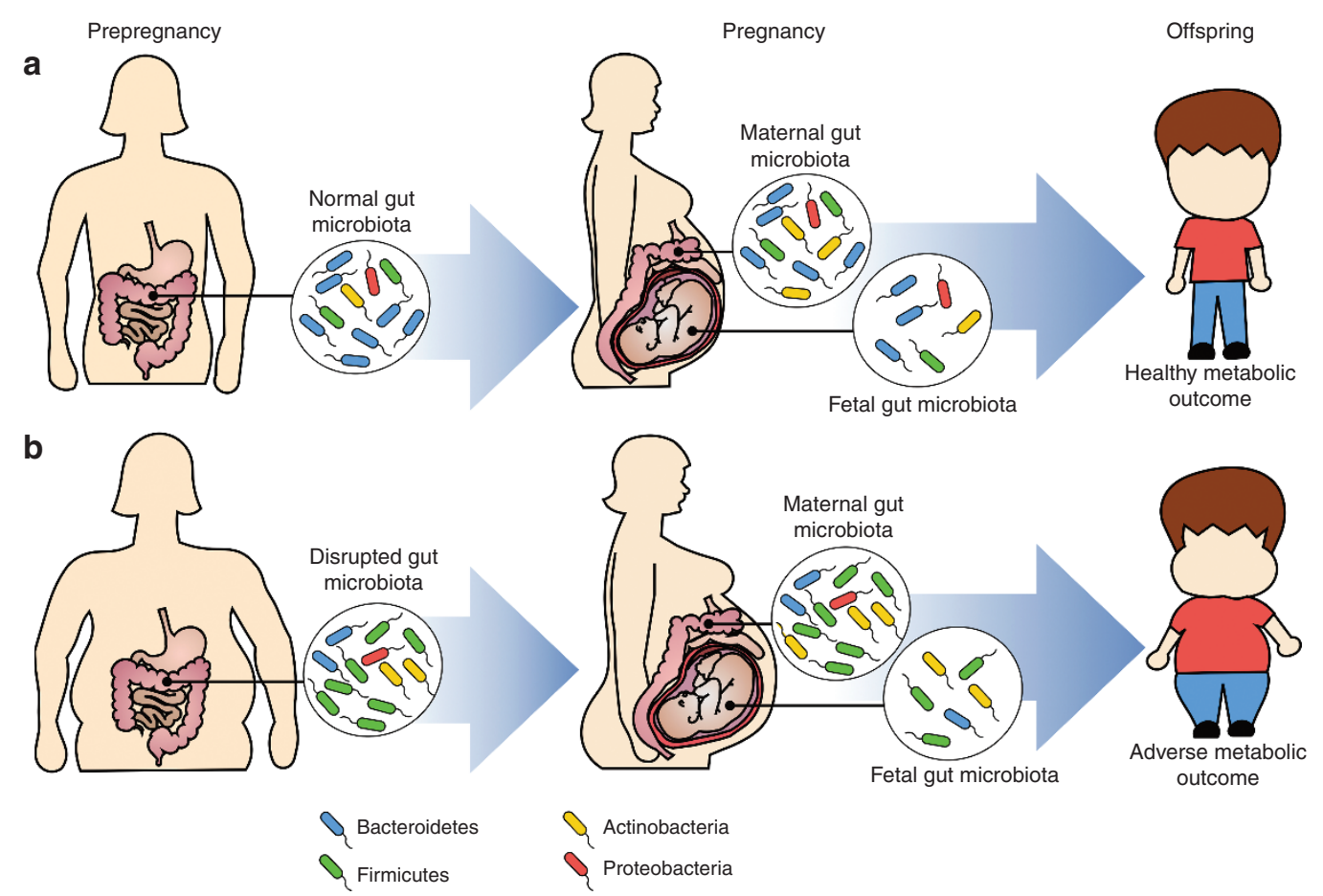

Figure 1. Overview of maternal gut microbiome modulation with pregnancy \pm obesity. Maternal gut microbiota composition changes over the course of pregnancy. It is proposed that a lean woman possesses a stable, healthy gut microbiota that is modulated over the course of pregnancy (a). How that is transferred to the fetus in utero, is still unknown, but may facilitate normal gut development and metabolic function in the offspring. Obese women (b) likely present with a disrupted gut microbiota already before pregnancy, and this is either amplified or further modified with pregnancy adaptations. These modifications lead to aberrant intrauterine environment that could lead to fetal poor/altered gut development and mediated increased chronic disease risk. Adapted with permission from ref. (58). 


\section{Review | Gohiretal.}

and BMI influence maternal and infant gut microbiota (67) and maternal obesity is associated with increased maternal, placental, and fetal inflammation. Maternal infection results in the translocation of bacterial fragments to the placenta (68), but if this occurs in pregnancies complicated by obesity is unknown.

\section{FETAL GUT MICROBIOME ACQUISITION IN UTERO}

Historically, fetal gut colonization was believed to initiate right before birth when the amniotic sac ruptures; prior to this point the fetus was thought to develop in a sterile environment within the amniotic sac. Over the past decade, numerous studies have provided evidence against this widely accepted dogma. The presence of bacteria in the placenta (69), fetal membranes (70), amniotic fluid (71), umbilical cord blood (71), and meconium (72) suggest maternal microbes are present in the intrauterine environment and within the intrauterine tissues. Consistent with this notion, the distinct microbiome signature of the meconium of newborn infants has been shown to be altered by maternal metabolic and health status (73). The presence of specific strains of maternal enteric bacteria in the meconium implies that the fetus is exposed to microbes from the maternal gut in utero (74) and points to the maternal gut as a key player in the development of fetal gut microbiome. Commensal bacteria from the maternal gut have been isolated from umbilical cord blood of healthy neonates born by cesarean section (71) and oral bacteria have been found in the placenta and amniotic fluid (71) in healthy term pregnancies, and likely unrelated to intra-amniotic infection and preterm labor $(69,75)$. It is possible that maternal gut dysbiosis as a result of obesity, poor nutrition, or even stress, could ultimately affect the microbes the fetus is exposed to in utero, and may serve to alter fetal gut development. It is this change in fetal gut microbiome composition (and ultimately gut development) that may potentially lead to changes in long-term gut function and lead to metabolic compromise.

Although data are very limited and at this stage speculative, it remains possible that bacterial contact in utero initiates fetal gut colonization. Gosalbes et al. found meconium microbiota was distinctly different in composition from the maternal vaginal and fecal microbiota (73). Two distinct types of meconium microbiota composition were characterized, one dominated by enteric bacteria and the second dominated by lactic acid bacteria. Maternal education level tended to be associated with meconium type and meconium diversity was modulated by maternal diabetes status (73), consistent with an observed increase in bacterial diversity if the mothers had pregestational diabetes, compared with gestational diabetes or no diabetes (76). Most recently, it has been shown that the placenta harbors a microbiome more similar to that of the non-pregnant human oral microbiome (75). These data support other studies documenting the presence of Gram-negative bacteria in the placental samples in the absence of infection (77), and support the notion that the fetal gut maybe colonized within the intrauterine milieu. However, it is important to note that the presence of bacterial DNA is not the same as live bacteria; and thus far live commensal bacterial populations have not been demonstrated to be present within the placenta. Furthermore, the source of these microbes that may exist in the placenta is unclear; although Aagaard et al. suggest that the maternal oral cavity may contribute to systemic bacterial counts that localize within the placenta possibly during implantation (75). Nevertheless the concept of maternal-offspring microbial transmission is well established in other animals (78) and thus it is likely that in humans maternal health and lifestyle can impact on maternal microbial transmission and the development of the fetal gut microflora diversity.

The mechanism by which maternal microbes enter the fetoplacental interface and come in contact with the fetus is not fully understood. It appears that maternal immune cells such as dendritic cells may play a role in maternal enteric bacteria translocation (79). Dendritic cells make contact with the paracellular space between the intestinal epithelial cells, opening up tight junctions located there (79). This process is aided by tight junction proteins such as occludin allowing dendritic cells to form new tight junction-like structures with the gut epithelial cells and infiltrating dendritic cells then directly sample bacteria from the gut lumen (79) and become internalized. The internalized intestinal commensal bacteria are viable for several days (80). Other immune cells such as leukocytes and neutrophils can also infiltrate the gut lumen using a similar mechanism (81). Although still speculative, it is possible that since these immune cells are migratory, internalized intestinal bacteria can be transported to different organ systems, one of them being the placenta via the circulation.

Changes in maternal intestinal permeability are likely to play a role in bacterial translocation. Little is known regarding maternal gut permeability during pregnancy and whether there are gestational-associated changes in gut leakage of bacterial fragments. Both paracellular and transcellular transport pathways participate in intestinal permeability. Paracellular pathways are defined by a number of proteins, although is mainly restricted by tight junctions including the claudins, occludins, and junctional adhesion molecule A (82). There is some evidence that the intestinal barrier changes during pregnancy compared with the non-pregnant state (83) but is an area that is significantly under investigated.

\section{THE INTRAUTERINE ENVIRONMENT: IMPACTS ON FETAL GUT DEVELOPMENT}

There is a growing body of research suggesting that maternal microbiota, prepregnancy and during pregnancy, and factors impacting it have a huge effect on the fetal gut. Animal studies have shown that manipulation of the maternal microbiota, either through antibiotics or probiotics, have consequences on the structural and functional development of the gut in their offspring. In a landmark study (84), pregnant rats were administered either broad-spectrum antibiotics $3 \mathrm{~d}$ before parturition or live non-pathogenic E. coli 1 wk before parturition and during lactation. The offspring were examined at $2 \mathrm{wk}$ of age and compared with the offspring of untreated mothers. Rat pups from antibiotic-treated mothers had decreased stomach 


\section{Maternal obesity and gut microbiome}

growth and gastric acid secretion as well as increased intestinal permeability; the rat pups from E. coli-treated mothers had similar decreased gastric acid secretion but had increased small intestinal growth as well as increased activity of key digestive enzymes (84). Offspring from both the antibiotictreated and E. coli-treated mothers had increased numbers of Enterobacteriaceae in their cecal contents (84). In a more recent study (85), pregnant sows were administered amoxicillin $10 \mathrm{~d}$ before and $21 \mathrm{~d}$ following parturition resulting in significant changes in the architecture of the small intestinal mucosa, with wider crypts, decreased absorption, and altered activity of mucosal enzymes. The composition of the intestinal microbiota was found to be altered in the offspring of the antibiotic-treated sows, with decreased relative abundance of presumed beneficial bacteria and increased abundance of presumed pathogenic bacterial populations (85).

Several studies have investigated the effects of probiotics during pregnancy on maternal and consequently, fetal gut microbiota colonization. Schultz et al. showed that infants of mothers who were taking Lactobacillus rhamnosus strain GG (L. GG) were colonized with $L$. GG for up to 12 mo after birth (86). Similar results for maternal to infant transmission of probiotics is seen in animal models of mice, rats, and sows (87). Probiotics taken by the mother during pregnancy appear to colonize the fetal gut-however-how long this colonization persists varies between subjects. Further evidence of maternal to fetal transmission of bacterial microbes comes from studies investigating the transmission of specific maternal enteric bacteria to the fetal gut. Makino et al. showed that specific Bifidobacterium strains found predominantly in the maternal intestines are stably colonized in infants for up to $90 \mathrm{~d}$ post birth (88).

Together, these studies strongly support the concept that changes in the maternal microbial environment can result in developmental abnormalities in the fetal intestine, thus putting the offspring at risk for life-long diseases of altered intestinal function.

\section{MATERNAL NUTRITIONAL IMPACTS ON THE GUT}

\section{MICROBIOME: MATERNAL AND OFFSPRING OUTCOMES}

Manipulation of maternal nutrition during pregnancy and prepregnancy has been shown to directly impact fetal growth and development. There is ample evidence supporting the notion that changes in maternal nutrition result in an adverse intrauterine environment that modulate fetal adaptations. At one end of the spectrum, intrauterine growth restriction induced by isocaloric protein restriction during pregnancy causes intestinal growth retardation in animal models $(89,90)$. Intrauterine growth restriction piglets have longer and thinner intestines at birth and seem to have delayed postnatal intestinal development (89). In rats, intrauterine growth restriction alters neonatal gut microbiota density where increased bacterial density occurs at postnatal day 5 and decreased bacterial density at day 12 . The opposite is observed in control pups, where gut microbiota density increases from postnatal day 5-12 and then stabilizes (90). It appears that not only does structure relate to fetal growth, but gut microbiota composition is also affected where growth restricted pups demonstrate reduced number of Bifidobacterium, the dominant species in infant and adult intestine.

On the other end of the nutritional spectrum, an adverse intrauterine environment caused by maternal HF diet intake has also been shown to impair fetal gut development. Myles et $a l$. investigated the effects of parental fat consumption during gestation and lactation on offspring microbiome and immune system (91), by placing breeding pairs on a special Western diet, containing $40 \%$ calories derived from omega 6 fatty acids $1 \mathrm{~d}$ before breeding. The offspring of Western diet breeders had significantly increased Firmicutes-to-Bacteroidetes ratio compared with control offspring and demonstrated an altered immune response (91). Due to the coprophagic nature of mice, the researchers cohoused offspring from the two groups in order to transfer microbiota between the groups of mice and observe whether the changes in immune response are mediated by the gut microbiota. Cohousing offspring of Western diet breeders and control resulted in no differences between the two groups in gut microbiota content, diversity, or immune response (91). This suggests that the altered immune response in Western diet offspring was associated with changes in their gut microbiota and can be reversed by colonization of "control" microbiota. Recently, Ma et al. studied the effects of maternal HF diet on the maternal and offspring microbiome of Macaca fuscata. Maternal HF diet altered the intestinal microbiome in the mothers and offspring. Gestational exposure to HF diet resulted in gut dysbiosis despite control diet post weaning in juvenile primates and was positively correlated with pathways involved in amino acid, carbohydrate, and lipid metabolism (92). The data support the idea that microbiome of offspring of mothers fed a HF diet has increased capacity to harvest energy from the diet.

Finally, it is important to consider the periconceptional environment. Although it has been well established in both clinical (93) and basic animal studies (4) that that periconceptual period is equally (and some might argue more) important in establishing long-term health and disease risk in offspring, no data currently exist describing the periconceptional maternal microbiome. Although it has been reported that first trimester pregnant microbiome is similar to that of the non-pregnant microbiome and shifts appear to occur predominantly in the third trimester $(57,58)$, it is unknown whether the periconceptional period presents a window of opportunity for microbial intervention. It is possible, however, that at least dietary interventions at this important time of embryonic and placental development could influence maternal microbial establishment and influence maternal adaptation to pregnancy and/ or fetal development. This is clearly an area that needs further exploration.

\section{CONCLUSIONS}

There strong evidence to suggest that host-bacterial relationships underlie some of the developmental adaptations that an organism makes early in life to accommodate its nutritional environment. Although this field of research is only at its 
infancy, it is exciting to consider role of pregnancy-associated changes in maternal gut microbiota composition influencing nutrient absorption, inflammation, microbial translocation as well as fetal gut colonization and metabolic tissue development in determining chronic disease risk. Gut microbial metabolism is modified by host inflammation; where the inflammatory host response creates a new metabolic niche, creating novel opportunities for potentially inferior microbes to thrive resulting in disruption of a balanced microbiota composition or dysbiosis (94). Whether an obesity-induced maternal pro-inflammatory environment is the result of, or the consequence of, fluctuating gut microbial populations is currently unclear. Whilst the maternal-placental-fetal unit ultimately dictates the growth and development of the fetus, we can no longer ignore the large population of bacteria that also inhabit the maternal environment and likely play a key role in maternal metabolic adaptation to pregnancy. These maternal microbiota are responsive to changes in their environment (within the gut) where changes in the mother's gut niche, be it inflammatory- or nutritionallymediated, may ultimately impact on maternal adaptation to pregnancy, the function of the placenta, and/or the growth of the fetus, and impart an increased risk of obesity and metabolic function in the offspring.

\section{STATEMENT OF FINANCIAL SUPPORT}

D.M.S. is supported by Canada Research Chairs Program; W.G. is supported by the Natural Sciences and Engineering Research Council (NSERC; Canada); and E.M.R. is supported by McMaster Children's Hospital.

Disclosure: The authors have no conflict of interest and nothing to disclose.

\section{REFERENCES}

1. World Health Organization. Obesity. 2013. (http://www.who.int/gho/ncd/ risk_factors/obesity_text/en/.)

2. Andreasen $\mathrm{CH}$, Andersen G. Gene-environment interactions and obesity-further aspects of genomewide association studies. Nutrition 2009;25:998-1003.

3. Gluckman PD, Hanson MA. Developmental plasticity and human disease: research directions. J Intern Med 2007;261:461-71.

4. Sinclair KD, Watkins AJ. Parental diet, pregnancy outcomes and offspring health: metabolic determinants in developing oocytes and embryos. Reprod Fertil Dev 2013;26:99-114.

5. Reynolds RM, Labad J, Buss C, Ghaemmaghami P, Räikkönen K. Transmitting biological effects of stress in utero: implications for mother and offspring. Psychoneuroendocrinology 2013;38:1843-9.

6. Thomas LV, Ockhuizen T, Suzuki K. Exploring the influence of the gut microbiota and probiotics on health: a symposium report. Br J Nutr 2014;112:Suppl 1:S1-18.

7. Nieuwdorp M, Gilijamse PW, Pai N, Kaplan LM. Role of the microbiome in energy regulation and metabolism. Gastroenterology 2014;146:1525-33.

8. Chow J, Lee SM, Shen Y, Khosravi A, Mazmanian SK. Host-bacterial symbiosis in health and disease. Adv Immunol 2010;107:243-74.

9. Bäckhed F, Ding H, Wang T, et al. The gut microbiota as an environmental factor that regulates fat storage. Proc Natl Acad Sci USA 2004;101:15718-23.

10. Turnbaugh PJ, Ley RE, Mahowald MA, Magrini V, Mardis ER, Gordon JI. An obesity-associated gut microbiome with increased capacity for energy harvest. Nature 2006;444:1027-31.

11. Ley RE, Turnbaugh PJ, Klein S, Gordon JI. Microbial ecology: human gut microbes associated with obesity. Nature 2006;444:1022-3.

12. Ley RE, Bäckhed F, Turnbaugh P, Lozupone CA, Knight RD, Gordon JI. Obesity alters gut microbial ecology. Proc Natl Acad Sci USA 2005;102:11070-5.
13. Turnbaugh PJ, Gordon JI. The core gut microbiome, energy balance and obesity. J Physiol 2009;587:4153-58.

14. Furet JP, Kong LC, Tap J, et al. Differential adaptation of human gut microbiota to bariatric surgery-induced weight loss: links with metabolic and low-grade inflammation markers. Diabetes 2010;59:3049-57.

15. Zhang H, DiBaise JK, Zuccolo A, et al. Human gut microbiota in obesity and after gastric bypass. Proc Natl Acad Sci USA 2009;106:2365-70.

16. Arumugam M, Raes J, Pelletier E, et al. Enterotypes of the human gut microbiome. Nature 2011;473:174-80.

17. Bergman EN. Energy contributions of volatile fatty acids from the gastrointestinal tract in various species. Physiol Rev 1990;70:567-90.

18. Payne AN, Chassard C, Zimmermann M, Müller P, Stinca S, Lacroix C. The metabolic activity of gut microbiota in obese children is increased compared with normal-weight children and exhibits more exhaustive substrate utilization. Nutr Diabetes 2011;1:e12.

19. Bervoets L, Van Hoorenbeeck K, Kortleven I, et al. Differences in gut microbiota composition between obese and lean children: a cross-sectional study. Gut Pathog 2013;5:10.

20. Harris K, Kassis A, Major G, Chou CJ. gut microbiota a new factor contributing to obesity and its metabolic disorders J Obes 2012;2012:879151.

21. Kim KA, Gu W, Lee IA, Joh EH, Kim DH. High fat diet-induced gut microbiota exacerbates inflammation and obesity in mice via the TLR4 signaling pathway. PLoS One 2012;7:e47713.

22. Turnbaugh PJ, Bäckhed F, Fulton L, Gordon JI. Diet-induced obesity is linked to marked but reversible alterations in the mouse distal gut microbiome. Cell Host Microbe 2008;3:213-23.

23. Zhang $\mathrm{C}$, Zhang $\mathrm{M}$, Wang $\mathrm{S}$, et al. Interactions between gut microbiota, host genetics and diet relevant to development of metabolic syndromes in mice. ISME J 2010;4:232.

24. Patro B, Liber A, Zalewski B, Poston L, Szajewska H, Koletzko B. Maternal and paternal body mass index and offspring obesity: a systematic review. Ann Nutr Metab 2013;63:32-41.

25. Kristensen J, Vestergaard M, Wisborg K, Kesmodel U, Secher NJ. Prepregnancy weight and the risk of stillbirth and neonatal death. BJOG 2005;112:403-8.

26. Triunfo S, Lanzone A. Impact of overweight and obesity on obstetric outcomes. J Endocrinol Invest 2014;37:323-9.

27. Wallace JM, Horgan GW, Bhattacharya S. Placental weight and efficiency in relation to maternal body mass index and the risk of pregnancy complications in women delivering singleton babies. Placenta 2012;33:611-8.

28. Priyadarshini M, Thomas A, Reisetter AC, et al. Maternal short-chain fatty acids are associated with metabolic parameters in mothers and newborns. Transl Res 2014;164:153-7.

29. Aye IL, Lager S, Ramirez VI, et al. Increasing maternal body mass index is associated with systemic inflammation in the mother and the activation of distinct placental inflammatory pathways. Biol Reprod 2014;90:129.

30. Aune D, Saugstad OD, Henriksen T, Tonstad S. Maternal body mass index and the risk of fetal death, stillbirth, and infant death: a systematic review and meta-analysis. JAMA 2014;311:1536-46.

31. Catalano PM, Presley L, Minium J, Hauguel-de Mouzon S. Fetuses of obese mothers develop insulin resistance in utero. Diabetes Care 2009;32:1076-80.

32. Stothard KJ, Tennant PW, Bell R, Rankin J. Maternal overweight and obesity and the risk of congenital anomalies: a systematic review and metaanalysis. JAMA 2009;301:636-50.

33. Challier JC, Basu S, Bintein T, et al. Obesity in pregnancy stimulates macrophage accumulation and inflammation in the placenta. Placenta 2008;29:274-81.

34. Farley DM, Choi J, Dudley DJ, et al. Placental amino acid transport and placental leptin resistance in pregnancies complicated by maternal obesity. Placenta 2010;31:718-24.

35. Aye IL, Jansson T, Powell TL. Interleukin- $1 \beta$ inhibits insulin signaling and prevents insulin-stimulated system A amino acid transport in primary human trophoblasts. Mol Cell Endocrinol 2013;381:46-55.

36. Malti N, Merzouk H, Merzouk SA, et al. Oxidative stress and maternal obesity: feto-placental unit interaction. Placenta 2014;35:411-6. 


\section{Maternal obesity and gut microbiome $\mid$ ReVieW}

37. Dubé E, Gravel A, Martin C, et al. Modulation of fatty acid transport and metabolism by maternal obesity in the human full-term placenta. Biol Reprod 2012;87:14, 1-11.

38. Sridhar SB, Darbinian J, Ehrlich SF, et al. Maternal gestational weight gain and offspring risk for childhood overweight or obesity. Am J Obstet Gynecol 2014;211:259.e1-8.

39. Sparano S, Ahrens W, De Henauw S, et al. Being macrosomic at birth is an independent predictor of overweight in children: results from the IDEFICS study. Matern Child Health J 2013;17:1373-81.

40. Aiken CE, Ozanne SE. Transgenerational developmental programming. Hum Reprod Update 2014;20:63-75.

41. Reynolds RM, Allan KM, Raja EA, et al. Maternal obesity during pregnancy and premature mortality from cardiovascular event in adult offspring: follow-up of 1323275 person years. BMJ 2013;347:f4539.

42. Williams L, Seki Y, Vuguin PM, Charron MJ. Animal models of in utero exposure to a high fat diet: a review. Biochim Biophys Acta 2014;1842:50719.

43. Howie GJ, Sloboda DM, Kamal T, Vickers MH. Maternal nutritional history predicts obesity in adult offspring independent of postnatal diet. J Physiol 2009;587(Pt 4):905-15.

44. Morris MJ, Chen H. Established maternal obesity in the rat reprograms hypothalamic appetite regulators and leptin signaling at birth. Int J Obes (Lond) 2009;33:115-22.

45. Elahi MM, Cagampang FR, Mukhtar D, Anthony FW, Ohri SK, Hanson MA. Long-term maternal high-fat feeding from weaning through pregnancy and lactation predisposes offspring to hypertension, raised plasma lipids and fatty liver in mice. Br J Nutr 2009;102:514-9.

46. Li M, Reynolds CM, Sloboda DM, Gray C, Vickers MH. Effects of taurine supplementation on hepatic markers of inflammation and lipid metabolism in mothers and offspring in the setting of maternal obesity. PLoS One 2013;8:e76961.

47. Gabory A, Roseboom TJ, Moore T, Moore LG, Junien C. Placental contribution to the origins of sexual dimorphism in health and diseases: sex chromosomes and epigenetics. Biol Sex Differ 2013;4:5.

48. Dahlhoff M, Pfister S, Blutke A, et al. Peri-conceptional obesogenic exposure induces sex-specific programming of disease susceptibilities in adult mouse offspring. BBA-Mol Basis Dis 2014;1842:304-17.

49. Gabory A, Ferry L, Fajardy I, et al. Maternal diets trigger sex-specific divergent trajectories of gene expression and epigenetic systems in mouse placenta. PLoS One 2012;7:e47986.

50. Walker SP, Ugoni AM, Lim R, Lappas M. Inverse relationship between gestational weight gain and glucose uptake in human placenta from female foetuses. Pediatr Obes 2014;9:e73-6.

51. Zhang L, Long NM, Hein SM, Ma Y, Nathanielsz PW, Ford SP. Maternal obesity in ewes results in reduced fetal pancreatic $\beta$-cell numbers in late gestation and decreased circulating insulin concentration at term. Domest Anim Endocrinol 2011;40:30-9.

52. Yan X, Huang Y, Zhao JX, et al. Maternal obesity-impaired insulin signaling in sheep and induced lipid accumulation and fibrosis in skeletal muscle of offspring. Biol Reprod 2011;85:172-8.

53. Lager S, Samulesson AM, Taylor PD, Poston L, Powell TL, Jansson T. Dietinduced obesity in mice reduces placental efficiency and inhibits placental mTOR signaling. Physiol Rep 2014;2:e00242.

54. Jones HN, Woollett LA, Barbour N, Prasad PD, Powell TL, Jansson T. High-fat diet before and during pregnancy causes marked up-regulation of placental nutrient transport and fetal overgrowth in C57/BL6 mice. FASEB J 2009;23:271-8.

55. Zhu MJ, Ma Y, Long NM, Du M, Ford SP. Maternal obesity markedly increases placental fatty acid transporter expression and fetal blood triglycerides at midgestation in the ewe. Am J Physiol Regul Integr Comp Physiol 2010;299:R1224-31.

56. Zhu MJ, Du M, Nathanielsz PW, Ford SP. Maternal obesity up-regulates inflammatory signaling pathways and enhances cytokine expression in the mid-gestation sheep placenta. Placenta 2010;31:387-91.

57. Collado MC, Isolauri E, Laitinen K, Salminen S. Distinct composition of gut microbiota during pregnancy in overweight and normal-weight women. Am J Clin Nutr 2008;88:894-9.
58. Koren O, Goodrich JK, Cullender TC, et al. Host remodeling of the gut microbiome and metabolic changes during pregnancy. Cell 2012;150:470-80.

59. Mukhopadhya I, Hansen R, El-Omar EM, Hold GL. IBD-what role do Proteobacteria play? Nat Rev Gastroenterol Hepatol 2012;9:219-30.

60. Jost T, Lacroix C, Braegger C, Chassard C. Stability of the maternal gut microbiota during late pregnancy and early lactation. Curr Microbiol 2014;68:419-27.

61. Flint HJ, Scott KP, Louis P, Duncan SH. The role of the gut microbiota in nutrition and health. Nat Rev Gastroenterol Hepatol 2012;9:577-89.

62. Harris K, Kassis A, Major G, Chou CJ. Is the gut microbiota a new factor contributing to obesity and its metabolic disorders? J Obes 2012;2012:879151.

63. Santacruz A, Collado MC, García-Valdés L, et al. Gut microbiota composition is associated with body weight, weight gain and biochemical parameters in pregnant women. Br J Nutr 2010;104:83-92.

64. Puertollano E, Kolida S, Yaqoob P. Biological significance of short-chain fatty acid metabolism by the intestinal microbiome. Curr Opin Clin Nutr Metab Care 2014;17:139-44.

65. Laitinen K, Poussa T, Isolauri E; Nutrition, Allergy, Mucosal Immunology and Intestinal Microbiota Group. Probiotics and dietary counselling contribute to glucose regulation during and after pregnancy: a randomised controlled trial. Br J Nutr 2009;101:1679-87.

66. Luoto R, Laitinen K, Nermes M, Isolauri E. Impact of maternal probioticsupplemented dietary counselling on pregnancy outcome and prenatal and postnatal growth: a double-blind, placebo-controlled study. Br J Nutr 2010;103:1792-9.

67. Collado MC, Isolauri E, Laitinen K, Salminen S. Effect of mother's weight on infant's microbiota acquisition, composition, and activity during early infancy: a prospective follow-up study initiated in early pregnancy. Am J Clin Nutr 2010;92:1023-30.

68. Lin D, Smith MA, Elter J, et al. Porphyromonas gingivalis infection in pregnant mice is associated with placental dissemination, an increase in the placental Th1/Th2 cytokine ratio, and fetal growth restriction. Infect Immun 2003;71:5163-8.

69. Pettker CM, Buhimschi IA, Magloire LK, Sfakianaki AK, Hamar BD, Buhimschi CS. Value of placental microbial evaluation in diagnosing intraamniotic infection. Obstet Gynecol 2007;109:739-49.

70. Steel JH, Malatos S, Kennea N, et al. Bacteria and inflammatory cells in fetal membranes do not always cause preterm labor. Pediatr Res 2005;57: 404-11.

71. Jiménez E, Fernández L, Marín ML, et al. Isolation of commensal bacteria from umbilical cord blood of healthy neonates born by cesarean section. Curr Microbiol 2005;51:270-4.

72. Jiménez E, Marín ML, Martín R, et al. Is meconium from healthy newborns actually sterile? Res Microbiol 2008;159:187-93.

73. Gosalbes MJ, Llop S, Vallès Y, Moya A, Ballester F, Francino MP. Meconium microbiota types dominated by lactic acid or enteric bacteria are differentially associated with maternal eczema and respiratory problems in infants. Clin Exp Allergy 2013;43:198-211.

74. Makino H, Kushiro A, Ishikawa E, et al. Transmission of intestinal Bifidobacterium longum subsp. longum strains from mother to infant, determined by multilocus sequencing typing and amplified fragment length polymorphism. Appl Environ Microbiol 2011;77:6788-93.

75. Aagaard K, Ma J, Antony KM, Ganu R, Petrosino J, Versalovic J. The placenta harbors a unique microbiome. Sci Transl Med 2014;6:237ra65.

76. Hu J, Nomura Y, Bashir A, et al. Diversified microbiota of meconium is affected by maternal diabetes status. PLoS One 2013;8:e78257.

77. Stout MJ, Conlon B, Landeau M, et al. Identification of intracellular bacteria in the basal plate of the human placenta in term and preterm gestations. Am J Obstet Gynecol 2013;208:226.e1-7.

78. Funkhouser LJ, Bordenstein SR. Mom knows best: the universality of maternal microbial transmission. PLoS Biol 2013;11:e1001631.

79. Rescigno M, Urbano M, Valzasina B, et al. Dendritic cells express tight junction proteins and penetrate gut epithelial monolayers to sample bacteria. Nat Immunol 2001;2:361-7.

80. Macpherson AJ, Uhr T. Induction of protective IgA by intestinal dendritic cells carrying commensal bacteria. Science 2004;303:1662-5. 
81. Rescigno M, Rotta G, Valzasina B, Ricciardi-Castagnoli P. Dendritic cells shuttle microbes across gut epithelial monolayers. Immunobiology 2001;204:572-81.

82. Ménard S, Cerf-Bensussan N, Heyman M. Multiple facets of intestinal permeability and epithelial handling of dietary antigens. Mucosal Immunol 2010;3:247-59.

83. Kerr CA, Grice DM, Tran CD, et al. Early life events influence whole-of-life metabolic health via gut microflora and gut permeability. Crit Rev Microbiol 2014;0:1-15.

84. Fåk F, Ahrné S, Molin G, Jeppsson B, Weström B. Microbial manipulation of the rat dam changes bacterial colonization and alters properties of the gut in her offspring. Am J Physiol Gastrointest Liver Physiol 2008;294: G148-54.

85. Arnal ME, Zhang J, Messori S, Bosi P, Smidt H, Lallès JP. Early changes in microbial colonization selectively modulate intestinal enzymes, but not inducible heat shock proteins in young adult swine. PLoS One 2014;9:e87967.

86. Schultz M, Göttl C, Young RJ, Iwen P, Vanderhoof JA. Administration of oral probiotic bacteria to pregnant women causes temporary infantile colonization. J Pediatr Gastroenterol Nutr 2004;38:293-7.

87. Buddington RK, Williams CH, Kostek BM, Buddington KK, Kullen MJ. Maternal-to-infant transmission of probiotics: concept validation in mice, rats, and pigs. Neonatology 2010;97:250-6.
88. Makino H, Kushiro A, Ishikawa E, et al. Mother-to-infant transmission of intestinal bifidobacterial strains has an impact on the early development of vaginally delivered infant's microbiota. PLoS One 2013;8: e78331.

89. D'Inca R, Gras-Le Guen C, Che L, Sangild PT, Le Huërou-Luron I. Intrauterine growth restriction delays feeding-induced gut adaptation in term newborn pigs. Neonatology 2011;99:208-16.

90. Fança-Berthon P, Hoebler C, Mouzet E, David A, Michel C. Intrauterine growth restriction not only modifies the cecocolonic microbiota in neonatal rats but also affects its activity in young adult rats. J Pediatr Gastroenterol Nutr 2010;51:402-13.

91. Myles IA, Fontecilla NM, Janelsins BM, Vithayathil PJ, Segre JA, Datta SK. Parental dietary fat intake alters offspring microbiome and immunity. J Immunol 2013;191:3200-9.

92. Ma J, Prince AL, Bader D, et al. High-fat maternal diet during pregnancy persistently alters the offspring microbiome in a primate model. Nat Commun 2014;5:3889.

93. Cetin I, Berti C, Calabrese S. Role of micronutrients in the periconceptional period. Hum Reprod Update 2010;16:80-95.

94. Faber F, Bäumler AJ. The impact of intestinal inflammation on the nutritional environment of the gut microbiota. Lett 2014; pii: S01652478(14)00083-2. doi: 10.1016/j.imlet.2014.04.014. 